\title{
High-frequency polarization properties of southern Kühr sources
}

\author{
R. Ricci ${ }^{1,2}$, I. Prandoni ${ }^{3}$, C. Gruppioni ${ }^{4}$, R. J. Sault ${ }^{2}$, and G. De Zotti ${ }^{1,5}$ \\ 1 SISSA/ISAS, Via Beirut 2-4, 34014 Trieste, Italy \\ 2 Australia Telescope National Facility, CSIRO, PO Box 76, Epping, NSW 2121, Australia \\ 3 Istituto di Radioastronomia, CNR, Via Gobetti 101, 40129, Bologna, Italy \\ ${ }^{4}$ INAF, Osservatorio Astronomico di Bologna, Via Ranzani 1, 40126 Bologna, Italy \\ 5 INAF, Osservatorio Astronomico di Padova, Vicolo dell'Osservatorio 5, 35122 Padova, Italy \\ e-mail: dezotti@pd.astro.it
}

Received 5 August 2003 / Accepted 21 November 2003

\begin{abstract}
We have observed 250 of the 258 southern sources in the complete $5 \mathrm{GHz} 1 \mathrm{Jy}$ sample by Kühr et al. (1981) using the Australia Telescope Compact Array (ATCA) at 18.5 GHz. This paper focuses on the polarization properties of this sample, while other properties will be addressed in a future paper. The analysis subdivides the sample into flat and steep spectrum sources using the classification of Stickel et al. (1994), where spectral indices were measured between 2.7 and $5 \mathrm{GHz}$. The polarized flux has been measured with a $S / N>5$ for 170 sources (114 flat-spectrum and 56 steep-spectrum). Upper limits have been set for additional 27 sources (12 flat-spectrum and 15 steep-spectrum). The median fractional polarization at $18.5 \mathrm{GHz}$ for the flat-spectrum sub-sample is $\Pi_{18.5} \simeq 2.7 \%$, which is about a factor of 2 higher than at $1.4 \mathrm{GHz}\left(\Pi_{1.4} \simeq 1.4 \%\right.$, based on NVSS data). No evidence was found of significant correlations of the fractional polarization with other source properties. The median value of $\Pi_{18.5}$ for the steep spectrum sources is $\simeq 4.8 \%$, but our sample might be biased against extended sources. We find some correlation between $\Pi_{18.5}$ and both the low-frequency $(1.4-5 \mathrm{GHz})$ and the high-frequency $(5-18.5 \mathrm{GHz})$ spectral indices. An important application of this work is in estimating the contamination of CMB polarization maps by extragalactic radio sources. Our results indicate that such contamination is within that estimated by Mesa et al. (2002).
\end{abstract}

Key words. radio continuum: galaxies - galaxies: nuclei - quasars: general - BL Lacertae objects: general - surveys

\section{Introduction}

Measurements of polarization properties of extragalactic radio sources provide unique information on magnetic field structure. The fractional polarization is a measure of the orderedness of the magnetic field in the emitting region. If synchrotron self-absorption and Faraday rotation can be neglected, the position angle will be orthogonal to the magnetic field orientation. Given this, it is surprising that high radio frequency polarimetric surveys of complete samples of extragalactic radio sources are so rare.

The general high-frequency polarization properties of radio sources are poorly known. Multifrequency observations by Jones et al. (1985) and Rudnick et al. (1985) of 20 flat-spectrum sources $\left(\alpha<0.5 ; S \propto v^{-\alpha}\right)$ showed that the median value of polarization is $\sim 2.5 \%$ in the range $1.4-90 \mathrm{GHz}$, independent of frequency. This would suggest that the general polarization properties can be accounted for by largely random magnetic fields. Note, however, that Jones et al. and Rudnick et al. stressed that their sample is not complete and selection criteria may introduce unpredictable biases. On the other hand, the relatively constant polarization fraction suggests that differential

Send offprint requests to: $\mathrm{R}$. Ricci, e-mail: ricci@sissa.it
Faraday rotation, or decreased contribution of relatively unpolarized opaque regions, or greater magnetic field ordering on the smaller scales dominating at millimeter wavelengths, may play minor roles (see also Saikia \& Salter 1988). Okudaira et al. (1993) made linear polarization observations at $10 \mathrm{GHz}$ for a complete sample of 93 flat-spectrum sources selected at $5 \mathrm{GHz}$ $\left(S_{5 \mathrm{GHz}}>0.8 \mathrm{Jy}\right)$. Okudaira et al. do not provide an average value for the fractional polarization at $10 \mathrm{GHz}$. From their tabulated source values one could infer a median fractional polarization of $2.3 \%$. On the other hand, Aller et al. (1999) find that the fractional linear polarization of a incomplete sample of $41 \mathrm{BL}$ Lac objects is generally higher at $14.5 \mathrm{GHz}$ (median value $5 \%$ ) than at $4.8 \mathrm{GHz}$ (median value $3.6 \%$ ). Also, the millimeter/sub-millimeter polarization survey of 26 blazars by Nartallo et al. (1998) yielded a median fractional polarization at $0.8-1.1 \mathrm{~mm}$ of $7.1 \%$, while the NVSS data indicate, for the same objects, a median fractional polarization of $\simeq 2 \%$ at $1.4 \mathrm{GHz}$ (Mesa et al. 2002). For a sample of steepspectrum sources with multifrequency polarimetric observations by Klein et al. (2003), Mesa et al. (2002) find a steady increase with frequency of the mean fractional polarization from $\simeq 3 \%$ at $1.4 \mathrm{GHz}$ to $8.65 \%$ at $10.6 \mathrm{GHz}$. It should be noted, however, that the sample is biased towards high polarizations as it 
includes only sources which have been detected in polarization at $10.6 \mathrm{GHz}$. The same effect may occur in the high redshift radio galaxy sample observed by Pentericci et al. (2000) at high resolution $(0.2)$, where typical fractional polarizations of $10-$ $20 \%$ at $8.4 \mathrm{GHz}$ were found.

A good understanding of the high-frequency polarization properties of extragalactic sources is crucial in analysing the polarization of the cosmic microwave background (CMB). This has been detected recently by the DASI experiment at $30 \mathrm{GHz}$ (Kovac et al. 2002) and by the WMAP satellite (Kogut et al. 2003) in several bands in the range 23-94 GHz. The extraction of the very weak $\mathrm{CMB}$ polarization signal requires both high sensitivity and careful control of foregrounds. In the frequency range relevant to these experiments, the polarized emission of extragalactic radio sources is expected to be the main contaminant on small angular scales (Sazhin \& Korolëv 1985; De Zotti et al. 1999).

In this paper we describe the results of linear polarization measurements at $18.5 \mathrm{GHz}$ of 250 of the 258 southern $\left(\delta<0^{\circ}\right)$ extragalactic sources in the $5 \mathrm{GHz}$ Kühr et al. (1981) all-sky sample. This sample is complete to $S_{5 \mathrm{GHz}} \geq 1 \mathrm{Jy}$, and contains 527 objects. This is, by far, the largest complete sample of radio sources for which high-frequency polarization measurements have been carried out. Observations and data reduction are described in Sects. 2 and 3, respectively. The results are analyzed and discussed in Sect. 4. In Sect. 5 we summarize our main conclusions.

\section{Observations}

Measurements were carried out with the Australia Telescope Compact Array (ATCA). Since 2001 this facility has been undergoing a major millimeter upgrade that will provide $12 \mathrm{~mm}$ and $3 \mathrm{~mm}$ receivers on all the ATCA antennas. In the commissioning phase, prototype $12 \mathrm{~mm}$ receivers were mounted on antennas CA02, CA03 and CA04. We took snapshot observations of the southern sources in the so-called EW367 array configuration. The two frequency bands were centered at 18.5 and $18.65 \mathrm{GHz}$ with each band having a bandwidth of $128 \mathrm{MHz}$. The array configuration that we used is quite compact: the longest baseline was 214.3 meters, giving a beamwidth of 15.6 arcsec.

The observations were performed in three runs during March 2002. The total observing time was 48 hours: 24 hours on March 20-21, 10 hours on March 21-22 and 14 hours on March 27-28. We used the "mosaic observing mode" because this allowed us to organize the observing sequence to minimize the slew time between the sources. The 258 sources were split in 36 clusters of sources. Clusters were observed four times each to produce a good spread in hour angles.

Many of these sources had already been targeted with the ATCA as part of a search for potential $22 \mathrm{GHz}$ calibrators. The $22 \mathrm{GHz}$ total intensity measurements with ATCA of the full sample were completed in January 2001; the results will be reported in a subsequent paper. These measurements were used to determine the integration time for each target source to ensure a detection at $\geq 5 \sigma$ for source polarizations $\geq 1 \%$. Nevertheless, because of limited observing time, we set an upper limit of
30 min per source. This means that for 43 sources with $22 \mathrm{GHz}$ flux density $\leq 0.192 \mathrm{Jy}$, the detection threshold is $\geq 1 \%$. Because of technical problems during the runs, 8 sources were not successfully observed.

\section{Data reduction}

The radio source 1934-638 was used as the primary flux density calibrator, while phase and gain calibrators were chosen from the ATCA calibrator list. The instrumental polarization was calibrated using measurements of Jupiter, which we assumed to be unpolarized.

Unfortunately the atmospheric phase stability during the observations was poor, so it was not possible to image the sources. Instead we decided to use non-imaging model-fitting techniques. To measure calibrators sufficiently frequently to correct for phase instability would have been quite expensive in time: this would have reduced significantly the number of sources that we could observe in the scheduled time.

The data were reduced in the MIRIAD software package (Sault et al. 1995) to derive source flux densities in all the Stokes parameters $(I, Q, U, V)$. In particular we used the MIRIAD task CALRED to determine the flux densities. In processing the visibilities, CALRED assumes a point source model, and produces a flux density estimate for each Stokes parameter for each scan. Along with the flux density and its error, the task computes out the theoretical rms noise, and a confusion parameter, $C$. The confusion parameter is an estimate of the fraction of the flux density that fails to conform to a point-source model.

A high value of $C$ means that the source is poorly modelled as a point and that the determination of its flux density is poor. On the other hand, even in the case of truly point sources (where the value of $C$ should be 0 ), noise results in a spread of the estimated value of $C$. For normal error distribution, $C$ is approximately described by a semi-Gaussian distribution (negative values of $C$ are not allowed). The observed distribution of $C$ (Fig. 1) shows a strong excess over the best-fit Gaussian curve where $C \geq 5 \%$ : we believe this is a result of source extension. Consequently we have chosen to exclude all the scans with $C \geq 5 \%$. This resulted in the rejection of 53 sources.

For the remaining 197 sources we computed the linearly polarized flux for each scan $i, F_{\mathrm{p}, i}=\sqrt{Q_{i}^{2}+U_{i}^{2}}$ and the associated error $\sigma_{\mathrm{F}, i}=\sigma_{Q, i}=\sigma_{U, i}\left(\sigma_{Q, U}\right.$ being the rms noise on the Stokes parameter $Q$ or $U$ ). For all the sources the polarized flux, $F_{\mathrm{p}}$, and its error, $\sigma_{\mathrm{F}}$, were obtained by using a weighted mean over all the scans:

$$
\begin{aligned}
& F_{\mathrm{p}}=\sigma_{\mathrm{F}}^{2} \sum_{i} \frac{F_{\mathrm{p}, i}}{\sigma_{\mathrm{F}, i}^{2}} \\
& \frac{1}{\sigma_{\mathrm{F}}^{2}}=\sum_{i} \frac{1}{\sigma_{\mathrm{F}, i}^{2}}
\end{aligned}
$$

For 170 sources the polarized flux was detected with a signalto-(theoretical)noise ratio $\geq 5$. We considered such values as reliable. An upper limit of $5 \times$ the theoretical noise was assigned to the other 27 sources. In all cases, the total intensity, 


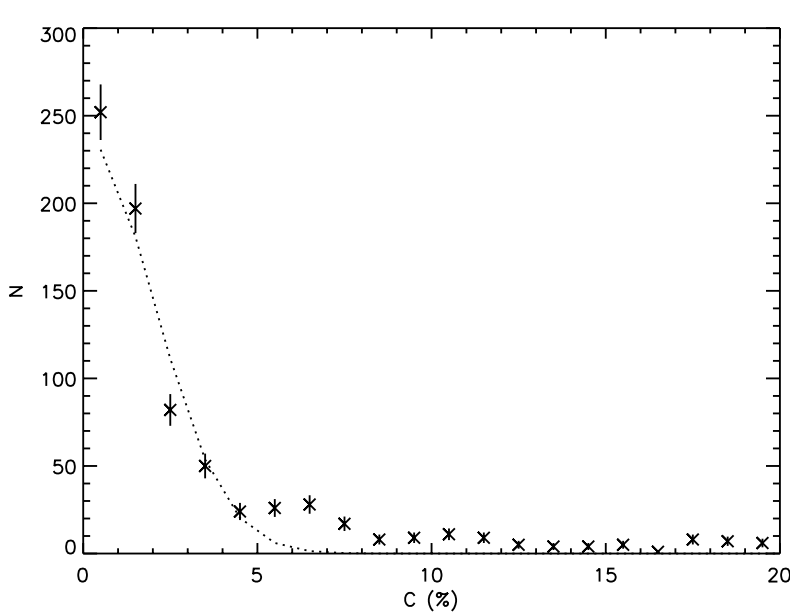

Fig. 1. Distribution of the confusion parameter $C$. A semi-Gaussian fit is also shown (dotted line). The fitted standard deviation is $\sigma=2.03$.

Table 1. Source number statistics for the polarization observations. The fractions in brackets are the percentages of flat and steep spectrum sources.

\begin{tabular}{lrrrrr}
\hline \hline & All & \multicolumn{2}{c}{ Flat } & \multicolumn{2}{c}{ Steep } \\
\hline detections & 170 & 114 & $(80 \%)$ & 56 & $(49 \%)$ \\
upper limits & 27 & 12 & $(8 \%)$ & 15 & $(13 \%)$ \\
rejected & 53 & 13 & $(9 \%)$ & 40 & $(35 \%)$ \\
not observed & 8 & 4 & $(3 \%)$ & 4 & $(3 \%)$ \\
\hline total & 258 & 143 & & 115 & \\
\hline
\end{tabular}

$I$ (again derived as the weighted mean of the scan total intensities), was measured with very high $S / N$ ratio. The observations did not determine the polarization position angle: the geometry on the ATCA $12 \mathrm{~mm}$ receivers was not calibrated sufficiently well to give reliable position angle information.

In the following analysis we subdivide the sample into flat and steep-spectrum sources. Following the classification of Stickel et al. (1994), we define as flat the sources with spectral index between 2.7 and $5 \mathrm{GHz} \alpha_{2.7}^{5}<0.5\left(S_{v} \propto v^{-\alpha}\right)$. Sources with $\alpha_{2.7}^{5}>0.5$ are considered steep. In Table 1 we list the number (and the corresponding fraction of the total) of detected sources for the flat and steep-spectrum sub-samples. For both classes we also list the upper limits and the rejected sources $(C>5 \%)$. While the final flat-spectrum source sample is almost complete $(88 \%)$, only $49 \%$ of the steep-spectrum sources have reliable detections.

The total intensities and polarized fluxes for steep and flatspectrum sources are listed in Tables 5 and 6, respectively. Also listed are the linear fractional polarization $\Pi_{18.5}=F_{\mathrm{p}} / I$, the spectral indices between 1.4 and $5 \mathrm{GHz}\left(\alpha_{1.4}^{5}\right)$ and 5 and $18.5 \mathrm{GHz}\left(\alpha_{5}^{18.5}\right)$ and the $5 \mathrm{GHz}$ luminosity. Source types and redshifts are from Stickel et al. (1994).

\section{Polarization properties of sources}

\subsection{Steep-spectrum sources}

As expected, most of the rejected sources (40 out of 53 with $C>5 \%)$ are steep-spectrum. This is because steep-spectrum sources are more likely extended than flat-spectrum sources.

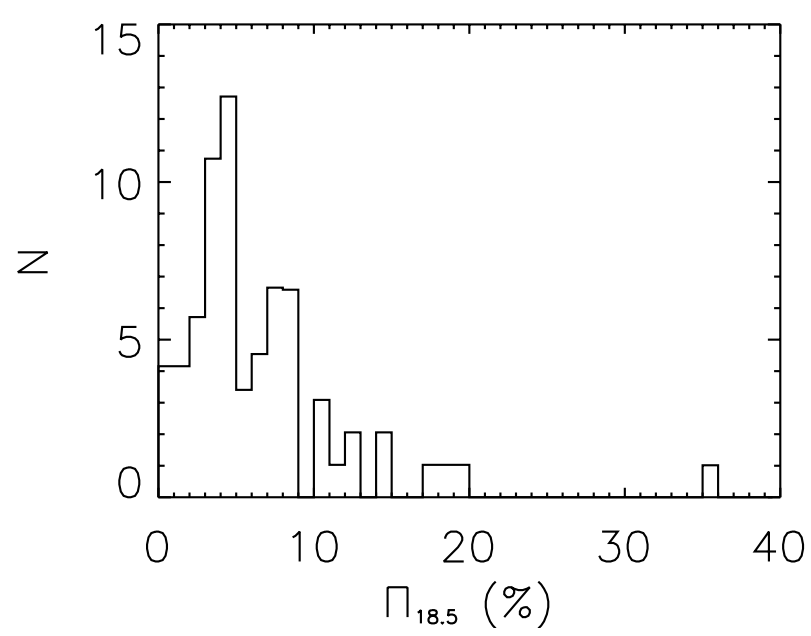

Fig. 2. Distribution of the fractional polarization at $18.5 \mathrm{GHz}$ for the steep-spectrum sources.

This means that the following analysis is based on an incomplete sample, which can be strongly biased against extended sources (see Table 1). Additionally, only upper limits can be given for 15 out of $71(\sim 21 \%)$ of the steep-spectrum sources. Nevertheless some points are worth noticing. In Fig. 2 we show the parent distribution of $\Pi_{18.5}$ for the steep-spectrum sample. This was obtained by using the Kaplan-Meier estimator (routine KMESTM in the software package ASURV Rev 1.2, (Isobe \& Feigelson 1990). This includes upper limits by using the survival analysis methods presented in Feigelson \& Nelson (1985) and Isobe et al. (1986)). The median of the distribution is $\simeq 4.8 \%$ and the mean is $\simeq 6.5 \pm 0.7 \%$. If we divide the sample into quasars (21 sources) and radio galaxies (50 sources) we find median values of $4.4 \%$ and $6.1 \%$, and mean values of $5.1 \pm 0.6 \%$ and $7.0 \pm 0.9 \%$ respectively. The Peto \& Prentice generalized Wilcoxon two sample test yields a probability of $21.7 \%$ that the distributions of QSOs and radio galaxies are drawn from the same parent population.

These values are somewhat lower than those found by Klein et al. (2003) at $10.6 \mathrm{GHz}$ and by Pentericci et al. (2000) at $8.4 \mathrm{GHz}$ by for their sample of high-redshift radio galaxies observed at high angular resolution (typical polarizations of 10$20 \%$ ). Nevertheless we point out that both the high spatial resolution of the Pentericci et al. sample and the selection criterion used by Klein et al. may introduce a bias toward high fractional polarization values, as stressed in the introduction.

We have used the NVSS public data (Condon et al. 1998) to investigate the polarization properties of our sources at 1.4 GHz. The 37 steep-spectrum sources detected by the NVSS in the overlapping region, have a median $1.4 \mathrm{GHz}$ fractional polarization of $\simeq 0.4 \%$, comparable to the residual instrumental polarization $(0.3 \%)$. To obtain a more reliable estimate of the $1.4 \mathrm{GHz}$ polarization, we included the steep-spectrum Kühr sources in the Northern sky. This selected 184 objects in total. We find $\Pi_{1.4 \text {,median }} \simeq 1.1 \%$. The Peto \& Prentice two-sample test rules out at a very high confidence level $\left(P<10^{-5}\right)$ the hypothesis that the distributions at 1.4 and $18.5 \mathrm{GHz}$ are drawn from the same parent population. 
Table 2. Test for correlations between $\Pi_{18.5}$ and other properties of the steep-spectrum sources. $N$ is the number of sources, $Z$ is the generalized Kendall's correlation coefficient and $P$ is the probability that a correlation is not present.

\begin{tabular}{lccc}
\hline \hline Correlation & $N$ & $Z$ & $P$ \\
\hline$\Pi_{18.5}-\alpha_{5}^{18.5}$ & 71 & 3.526 & 0.0004 \\
$\Pi_{18.5}-\alpha_{1.4}^{5}$ & 37 & 2.747 & 0.006 \\
$\Pi_{18.5}-z$ & 49 & 1.283 & 0.1996 \\
$\Pi_{18.5}-\log L_{5} \mathrm{GHz}$ & 27 & 0.764 & 0.445 \\
$\Pi_{18.5}-\Pi_{1.4}$ & 37 & 0.526 & 0.5992 \\
\hline
\end{tabular}

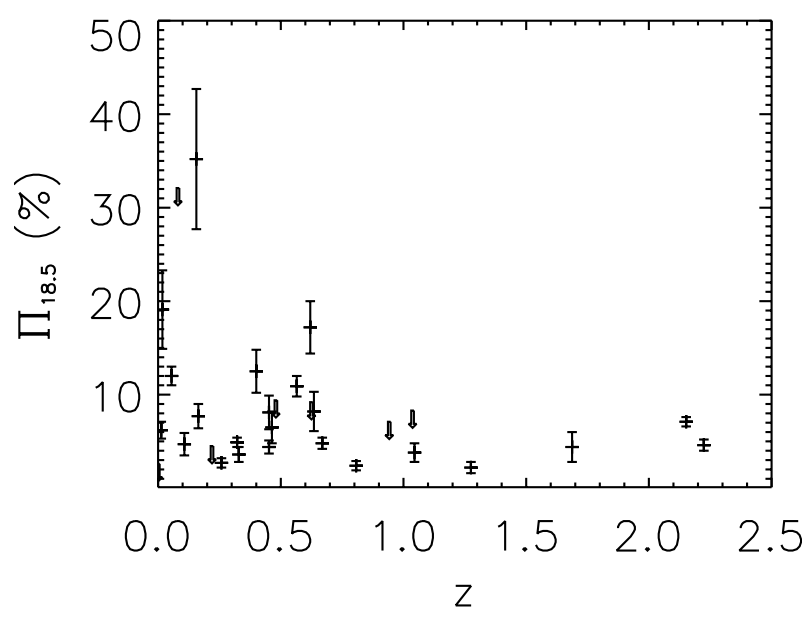

Fig. 3. $\Pi_{18.5}$ vs. redshift for the steep-spectrum sample. $\Pi_{18.5}$ upper limits are represented by downward arrows.

The origin of this frequency dependence is not well understood. The multifrequency polarization measurements by Klein et al. (2003), quoted by Mesa et al. (2002), indicated an increase by a factor of $\simeq 3$ of the fractional polarization of steepspectrum sources between 1.4 and $10.4 \mathrm{GHz}$. This is attributed to Faraday depolarization in uniform slabs with effective rotation measure $R M \simeq 260 \mathrm{rad} \mathrm{m}^{-2}$. However, an increase of the fractional polarization with frequency, as expected in the case of a Faraday screen at the source, translates in a positive correlation with redshift. For example, in the presence of a Faraday screen located at redshift $z$, the observed rotation measure is related to the intrinsic value by $R M_{\mathrm{obs}}=R M_{\mathrm{intr}}(1+z)^{-2}$. However no significant correlation is found in our data between $\Pi_{18.5}$ and $z$ (see Fig. 3 and Table 2). This may be consistent with the findings by Pentericci et al. (2000) that the fraction of powerful radio galaxies with extreme Faraday rotation measures $\left(R M>1000 \mathrm{rad} \mathrm{m}^{-2}\right)$ increases steeply with redshift. This suggests that their average environment becomes increasingly dense with increasing $z$. The larger mean fractional polarization measured at $18.5 \mathrm{GHz}$ may imply that Faraday depolarization is negligible at high frequencies. Polarization measurements at intermediate frequencies are necessary to assess this.

Table 2 also shows the results of our investigation of possible correlations of $\Pi_{18.5}$ with other source properties. There are indications of a correlation with the spectral indices $\alpha_{1.4}^{5}$ and (more significantly) $\alpha_{5}^{18.5}$ : steeper sources seem to have stronger $\Pi_{18.5}$ (Fig. 4). The fact that sources with the highest $\Pi_{18.5}$ and the steepest $\alpha_{5}^{18.5}$ are generally at low redshift may

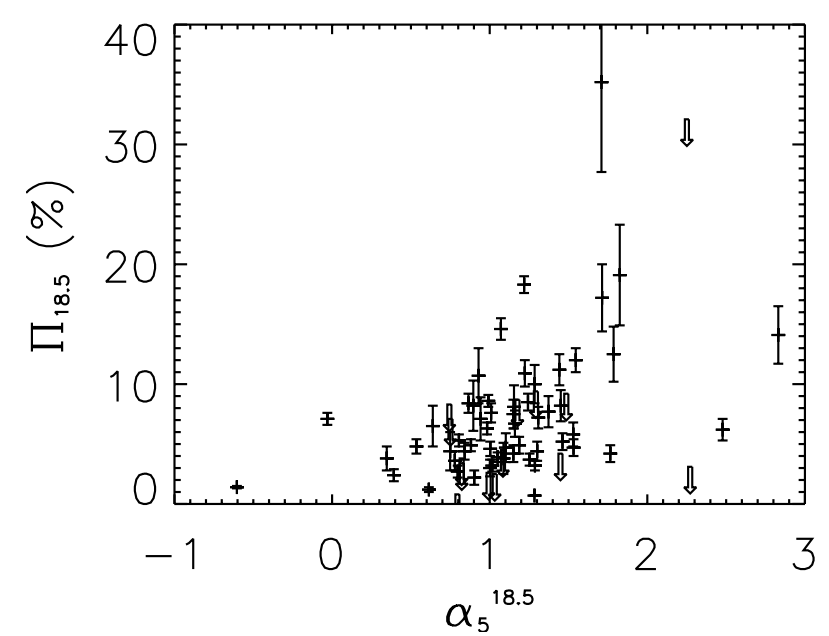

Fig. 4. $\Pi_{18.5}$ vs. $\alpha_{5}^{18.5}$ for the steep-spectrum sample. $\Pi_{18.5}$ upper limits are represented by downward arrows.



Fig. 5. Distribution of the fractional polarization at $18.5 \mathrm{GHz}$ for the flat-spectrum sample.

suggest that they are resolved and we are actually seeing highly polarized portions of them, such as radio lobes. We note, however, that any correlation should be taken with caution due to the incompleteness of the steep-spectrum source sample. The fractional polarizations at 18.5 and $1.4 \mathrm{GHz}$ are uncorrelated. We do not see any correlation between fractional polarization and other source properties.

\subsection{Flat-spectrum sources}

We used the Kaplan-Meyer estimator to derive the fractional polarization distribution of the 126 flat-spectrum sources in our final sample (114 detections and 12 upper limits). The result is shown in Fig. 5: the median value is $2.7 \%$ and the mean value is $2.9 \pm 0.1 \%$. This is about a factor of 2 lower than that found for the steep-spectrum sources.

We used the NVSS catalog (Condon et al. 1998) to determine the fractional polarization at $1.4 \mathrm{GHz}, \Pi_{1.4}: 86$ flatspectrum sources overlap with the NVSS survey. The distribution of $\Pi_{1.4}$ is shown in Fig. 6: it has a median value of $1.4 \%$, 


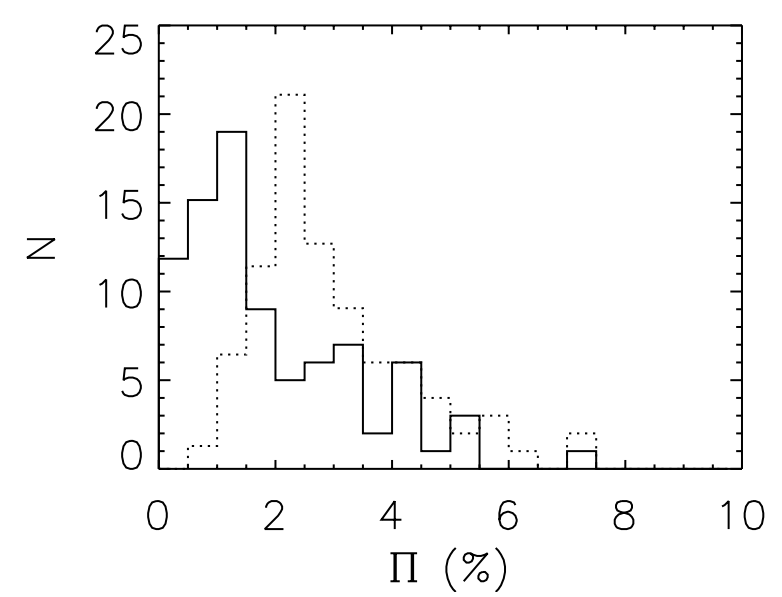

Fig. 6. Linear fractional polarization distribution at $1.4 \mathrm{GHz}$ (solid line) and $18.5 \mathrm{GHz}$ (dotted line) for the 86 flat-spectrum southern Kühr sources in the NVSS survey.

Table 3. Median fractional polarization of flat-spectrum sources. Columns 2 and 5 give the number and the median fractional polarization at $18.5 \mathrm{GHz}$ of the different classes of sources in our sample. In parentheses are the number of such sources in the NVSS area (Col. 3), and their median fractional polarization at $18.5 \mathrm{GHz}(\mathrm{Col} .6)$ and at $1.4 \mathrm{GHz}$ (Col. 7). Columns 4 and 8 give the total number of Kühr flatspectrum sources of the different classes in the NVSS catalog, and their median fractional polarization at $1.4 \mathrm{GHz}$.

\begin{tabular}{lrrrrrrr}
\hline \hline Type & \multicolumn{1}{c}{$N$} & \multicolumn{3}{c}{$\Pi_{18.5}(\%)$} & \multicolumn{2}{c}{$\Pi_{1.4}(\%)$} \\
\hline QSO & 108 & $(74)$ & 175 & 2.7 & $(2.6)$ & $(1.4)$ & 1.4 \\
BL Lac & 8 & $(5)$ & 34 & 3.0 & $(2.9)$ & $(1.2)$ & 2.2 \\
GAL & 8 & $(5)$ & 25 & 2.4 & $(2.5)$ & $(1.8)$ & 0.3 \\
\hline
\end{tabular}

comparable to the value found for steep-spectrum sources, and a factor of 2 lower than that at $18.5 \mathrm{GHz}$. This seems to be in disagreement with the results of Jones et al. (1985) and Rudnick et al. (1985), who found a constant polarization value of $2.5 \%$ in the frequency range $1.4-90 \mathrm{GHz}$.

In Table 3 we give the median fractional polarization for different classes of objects in the flat-spectrum sample (QSOs, BL Lacs and radio galaxies). In the last column of the table, we give the median fractional polarization at $1.4 \mathrm{GHz}$ that was computed by including the northern $(\delta>0)$ Kühr sources with NVSS detection. Adding the latter sources increases the total number of flat-spectrum sources with $1.4 \mathrm{GHz}$ polarization data from 86 to 238: the number of QSOs increases from 74 to 175 , that of BL Lacs from 5 to 34 , and that of galaxies from 5 to 25 . We do not find any significant indication of differences in the $18.5 \mathrm{GHz}$ polarization properties of the various sub-populations. However the very small number of BL Lacs and radio galaxies means that this comparison is not very meaningful. The comparison at $1.4 \mathrm{GHz}$ is more significant: the Peto \& Prentice generalized Wilcoxon two sample test yields probabilities of $\simeq 1 \%$ and of $0.001 \%$ that the fractional polarization distributions of QSOs are drawn from the same parent population as BL Lacs and radio galaxies, respectively.

As illustrated in Fig. 7, sources that are weakly polarized at $1.4 \mathrm{GHz}$ show a stronger fractional polarization at $18.5 \mathrm{GHz}$. This could be suggestive of differential Faraday depolarization



Fig. 7. $\Pi_{18.5}$ vs. $\Pi_{1.4}$ for the flat-spectrum sample. $\Pi_{18.5}$ upper limits are represented by downward arrows.

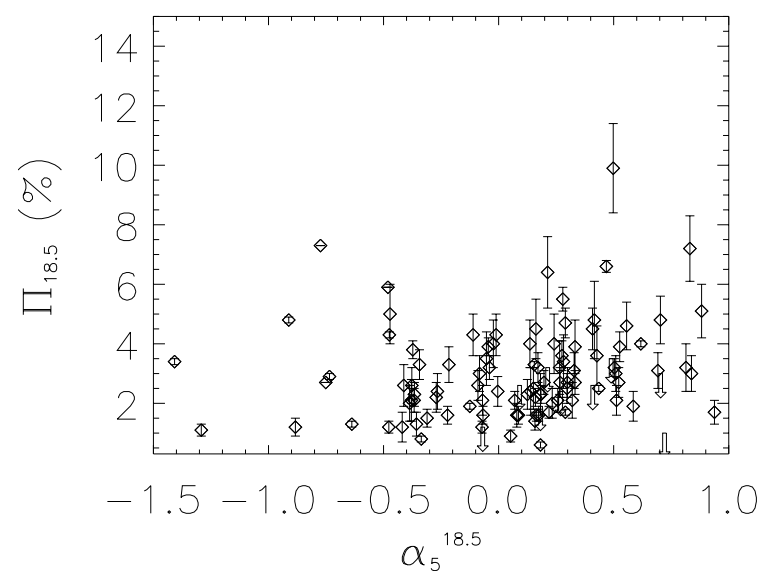

Fig. 8. $\Pi_{18.5}$ vs. $\alpha_{5}^{18.5}$ for the 108 flat-spectrum QSOs. $\Pi_{18.5}$ upper limits are indicated by downward arrows.

effects. Nevertheless, we do not see an increase of the fractional polarization with redshift, as might be expected in the case of Faraday depolarization inside the source. It should be remembered that the emission at different frequencies comes from different regions in the source, and that these regions may have intrinsically different polarization properties. On the other end, sources with $\Pi_{1.4} \gtrsim 2 \%$ tend to have $\Pi_{1.4} \gtrsim \Pi_{18.5}$, which may be the result of the decreased contribution, at high-frequency, of the more polarized, steep-spectrum, diffuse component.

To get further hints on mechanisms that determine the polarization properties of sources, we used the generalized Kendall's rank correlation coefficient $Z$ to look for possible correlations between $\Pi_{18.5}, \Pi_{1.4}$ and other properties of our flatspectrum radio sources, such as: the low-frequency spectral index (between $1.4 \mathrm{GHz}$ and $5 \mathrm{GHz}$ ), the high-frequency spectral index (between 5 and $18.5 \mathrm{GHz}$ ), the monochromatic luminosity at $5 \mathrm{GHz}$. The values of the probability $P$ that no correlation is present are given for both the whole flat-spectrum sample and the flat-spectrum QSOs only in Table 4. At variance with what found for the steep-spectrum sample, there is no significant evidence of a correlation between $\Pi_{18.5}$ and $\alpha_{5}^{18.5}$ (see Fig. 8). 
Table 4. Test for correlations between the fractional polarization at 18.5 and $1.4 \mathrm{GHz}$ and other properties of the flat-spectrum sources (whole sample and QSO sub-sample only). $Z$ is the generalized Kendall's correlation coefficient and $P$ is the probability that a correlation is not present.

\begin{tabular}{lcccc}
\hline \hline Correlation & \multicolumn{2}{c}{$Z$} & \multicolumn{2}{c}{$P$} \\
\hline & All & QSO & All & QSO \\
\hline$\Pi_{18.5}-\alpha_{1.4}^{5}$ & 0.47 & 0.23 & 0.48 & 0.63 \\
$\Pi_{18.5}-\alpha_{5}^{18.5}$ & 1.83 & 2.29 & 0.07 & 0.02 \\
$\Pi_{18.5}-z$ & 0.23 & 0.09 & 0.82 & 0.93 \\
$\Pi_{18.5}-L_{5 \mathrm{GHz}}$ & 0.91 & 0.56 & 0.36 & 0.58 \\
$\Pi_{18.5}-\Pi_{1.4}$ & 0.95 & 0.24 & 0.34 & 0.81 \\
\hline$\Pi_{1.4}-\alpha_{1.4}^{5}$ & 0.89 & 0.89 & 0.38 & 0.37 \\
$\Pi_{1.4}-\alpha_{5}^{18.5}$ & 1.90 & 1.47 & 0.05 & 0.14 \\
$\Pi_{1.4}-z$ & 1.85 & 1.53 & 0.06 & 0.13 \\
$\Pi_{1.4}-L_{5 \mathrm{GHz}}$ & 1.75 & 1.58 & 0.08 & 0.11 \\
\hline
\end{tabular}

\section{Discussion and conclusions}

We have performed high-frequency observations of a large, complete sample of bright extragalactic radio sources: 250 of the 258 sources in the southern sky $\left(\delta<0^{\circ}\right)$ of the $5 \mathrm{GHz}$ Kühr et al. (1981) sample were observed with the ATCA at $18.5 \mathrm{GHz}$. This sample is complete to $S_{5 \mathrm{GHz}} \geq 1 \mathrm{Jy}$. The polarized flux has been measured to better than $5 \sigma$ for 170 sources (114 flat-spectrum and 56 steep-spectrum) and upper limits have been set for additional 27 sources (12 flat-spectrum and 15 steep-spectrum). This represents to $88 \%$ of the flat-spectrum sources and $62 \%$ of the steep-spectrum sources in the southern Kühr sample. 53 sources were not considered in our analysis, as they appear to be extended (i.e. our point-source model fitting technique showed a significant fraction of non-pointlike emission). Most of the extended sources are steep-spectrum. Consequently our conclusions about this class of objects may be biased against extended sources.

The median fractional polarization at $18.5 \mathrm{GHz}$ for steep and flat-spectrum sources was found to be $\simeq 4.8 \%$ and $\simeq 2.7 \%$, respectively. The distributions are both skewed, with tails towards high values, up to a maximum of $\simeq 20 \%$ and $\simeq 10 \%$, respectively. That steep-spectrum sources generally display higher fractional polarization than flat-spectrum sources could be explained either in terms of different intrinsic polarization properties of the two classes of objects, or in terms of different internal depolarization effects (flat sources, usually dominated by their core emission, could be more heavily depolarized). An alternative to depolarization by internal Faraday rotation is also offered by gradients in an external Faraday screen that are not resolved by our observations. However, these two depolarizing mechanisms cannot be distinguished given polarization measurements at only two frequencies. Moreover, spectral indices and polarization levels of low redshift steep-spectrum radio sources with high polarization degrees $\left(\Pi_{18.5}>10 \%\right)$ could be overestimated due to resolution effects. Magnetic fields are more ordered on small angular scales, thus increasing polarization degrees on those scales.
We used the NVSS survey data to derive the fractional polarization at $1.4 \mathrm{GHz}$ for the Kühr all-sky sample. We find very similar median values for both the steep and the flat sources ( $1.1 \%$ and $1.4 \%$, respectively). This means a factor of $\sim 4(\sim 2)$ increase from 1.4 to $18.5 \mathrm{GHz}$ for the steep (flat) spectrum sources. It is unclear whether such an increase can be attributed to a decreased internal Faraday depolarization. In this case we would expect the fractional polarization to be correlated with redshift. However such a correlation is not found either for the steep or for the flat spectrum sources. For steep-spectrum sources, any redshift dependence could be counteracted by a steep increase of the intrinsic Faraday rotation measures with redshift, reflecting an increase of the environmental density (Pentericci et al. 2000). For the flat-spectrum sample, on the other hand, it is more likely that the increase in polarization is because the radio emission at different frequencies comes from different core components, that may have intrinsically different polarization properties.

We do not see any significant correlation between $\Pi_{18.5}$ and other source properties, such as the low-frequency spectral index and the luminosity at $5 \mathrm{GHz}$. A possible weak $(3 \sigma)$ correlation between $\Pi_{18.5}$ and the high-frequency spectral index, $\alpha_{5}^{18.5}$, is apparent in the steep-spectrum sample only.

An important application of these results is in the estimate of the contamination of CMB polarization maps by extragalactic radio sources (Mesa et al. 2002). Based on the multifrequency radio polarimetry by Klein et al. (2003), Mesa et al. (2002) estimated an average increase by a factor of 3 of the fractional polarization of steep-spectrum sources from $1.4 \mathrm{GHz}$ to higher frequencies. Our data suggest an increase by a larger factor $(\sim 4)$, although caution is needed because of the strong incompleteness of the steep-spectrum sample. On the other hand, it is worth noticing that steep-spectrum sources provide a minor contribution to polarization fluctuations at frequencies ( $\gtrsim 30 \mathrm{GHz}$ ) where $\mathrm{CMB}$ measurements are carried out. For flat-spectrum sources, Mesa et al. (2002) considered two possibilities: that the fractional polarization remains, on average, constant from 1.4 GHz to high frequencies (as found by Rudnick et al. 1985), or increases by a factor of 3 (as found by Nartallo et al. 1998). Our results are intermediate (an increase of a factor of $\sim 2$ ). This suggests a contamination of CMB polarization maps which is likely to be within the ranges considered by Mesa et al. (2002).

Acknowledgements. This research was supported in part by the Italian Space Agency (ASI) and by the Italian MIUR through a COFIN grant. RR gratefully acknowledges a financial contribution from the Italian National Research Council (CNR) as part of an exchange program with CSIRO; he also warmly thanks the Paul Wild Observatory staff for their kind hospitality at Narrabri (NSW, Australia) where some of this work was completed. We are grateful to the referee for useful comments that helped improving the paper. 
Table 5. Summary of observed and derived quantities for the steep spectrum sample: (1) source name (B1950); (2) observation dates $(1=$ March 20-21, 2 = March 22, 3 = March 27-28); (3) redshift; (4) source type; (5)-(6) $18.5 \mathrm{GHz}$ total intensity and its error in mJy; (7)-(8) 18.5 GHz polarized flux and its error in mJy; (9)-(10) $18.5 \mathrm{GHz}$ fractional polarization (\%) and its error (\%); (11) spectral index between 1.4 and $5 \mathrm{GHz}$; (12) spectral index between 5 and $18.5 \mathrm{GHz}$; (13) logarithm of the $5 \mathrm{GHz}$ luminosity in $\mathrm{W} \mathrm{Hz}^{-1}$; (14) $1.4 \mathrm{GHz}$ NVSS fractional polarization (\%).

\begin{tabular}{|c|c|c|c|c|c|c|c|c|c|c|c|c|c|}
\hline (1) & (2) & $\overline{~(3) ~}$ & (4) & (5) & (6) & $\overline{\overline{(7)}}$ & (8) & (9) & (10) & (11) & (12) & (13) & 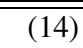 \\
\hline name & dates & $z$ & type & $I$ & $\sigma_{I}$ & $F_{\mathrm{p}}$ & $\sigma_{\mathrm{F}}$ & $\Pi_{18.5}$ & $\sigma_{\Pi}$ & $\alpha_{1.4}^{5}$ & $\alpha_{5}^{18.5}$ & $\log L_{5}$ & $\Pi_{1.4}$ \\
\hline $0003-003$ & 2,3 & 1.03700 & QSO & 525.0 & 14.0 & $<43.5$ & 8.7 & $<8.3$ & 1.7 & 0.81 & 0.74 & 27.86 & 0.66 \\
\hline $0008-421$ & 1 & $\ldots$ & $\mathrm{EF}$ & 181.6 & 0.6 & 9.4 & 1.2 & 5.2 & 0.7 & $\ldots$ & 1.46 & $\ldots$ & $\ldots$ \\
\hline $0022-423$ & 1 & $\ldots$ & QSO & 339.9 & 0.6 & 10.9 & 1.4 & 3.2 & 0.4 & $\ldots$ & 1.28 & $\ldots$ & $\ldots$ \\
\hline $0023-263$ & 3 & 0.32200 & GAL & 1143.7 & 5.5 & 55.8 & 6.2 & 4.9 & 0.5 & 0.69 & 0.88 & 27.20 & 0.11 \\
\hline $0035-024$ & 2,3 & 0.21970 & GAL & 651.0 & 14.0 & $<29.0$ & 5.8 & $<4.5$ & 0.9 & 0.65 & 1.08 & 26.73 & 0.20 \\
\hline 0039-445 & 1 & $\ldots$ & GAL & 218.6 & 0.7 & 15.7 & 2.0 & 7.2 & 0.9 & $\ldots$ & 1.30 & $\ldots$ & $\ldots$ \\
\hline $0042-357$ & 1 & $\ldots$ & NO ID & 269.9 & 0.7 & 20.6 & 2.1 & 7.6 & 0.8 & 0.73 & 1.00 & $\ldots$ & 1.31 \\
\hline $0045-255$ & 2 & 0.00086 & GAL & 638.4 & 2.9 & $<16.6$ & 3.3 & $<2.6$ & 0.5 & 0.19 & 0.99 & 21.84 & $<0.10$ \\
\hline $0105-163$ & 2,3 & 0.40000 & GAL & 114.0 & 2.0 & 14.2 & 2.6 & 12.5 & 2.3 & 0.99 & 1.78 & 26.95 & 0.45 \\
\hline 0114-211 & 2,3 & $\ldots$ & $\mathrm{EF}$ & 280.5 & 1.7 & 18.9 & 3.1 & 6.7 & 1.1 & 0.91 & 1.16 & $\ldots$ & 0.10 \\
\hline $0117-155$ & 2,3 & 0.56500 & GAL & 325.0 & 2.0 & 35.5 & 3.7 & 10.9 & 1.1 & 0.90 & 1.22 & 27.39 & 0.68 \\
\hline $0123-016$ & 2,3 & 0.01770 & GAL & 170.0 & 6.0 & 32.4 & 7.1 & 19.1 & 4.2 & $\ldots$ & 1.82 & $\ldots$ & $\ldots$ \\
\hline $0131-367$ & 1 & $\ldots$ & GAL & 54.9 & 0.6 & 7.8 & 1.3 & 14.1 & 2.4 & $\ldots$ & 2.83 & $\ldots$ & $\ldots$ \\
\hline $0159-117$ & 2,3 & 0.66900 & QSO & 690.0 & 5.0 & 33.1 & 4.4 & 4.8 & 0.6 & 0.53 & 0.53 & 27.40 & 3.52 \\
\hline $0201-440$ & 1 & $\ldots$ & QSO? & 256.0 & 1.4 & 37.3 & 2.4 & 14.6 & 0.9 & $\ldots$ & 1.07 & $\ldots$ & $\ldots$ \\
\hline 0235-197 & 2 & 0.62000 & GAL & 154.1 & 1.0 & 26.5 & 4.3 & 17.2 & 2.8 & 0.91 & 1.71 & 27.43 & 2.93 \\
\hline $0237-233$ & 2 & 2.22400 & QSO & 915.8 & 3.5 & 42.0 & 5.9 & 4.6 & 0.6 & 0.47 & 1.00 & 28.78 & 0.83 \\
\hline $0252-712$ & 1 & $\ldots$ & GAL & 316.6 & 0.9 & 11.7 & 1.7 & 3.7 & 0.5 & $\ldots$ & 1.25 & $\ldots$ & $\ldots$ \\
\hline $0407-658$ & 1 & $\ldots$ & QSO? & 462.0 & 2.2 & 21.7 & 3.0 & 4.7 & 0.7 & $\ldots$ & 1.53 & $\ldots$ & $\ldots$ \\
\hline $0409-752$ & 1 & $\ldots$ & GAL & 984.0 & 4.8 & 73.3 & 11.7 & 7.5 & 1.2 & $\ldots$ & 1.15 & $\ldots$ & $\ldots$ \\
\hline 0413-210 & 2 & 0.80700 & QSO & 856.1 & 2.3 & 20.4 & 4.2 & 2.4 & 0.5 & 0.52 & 0.39 & 27.57 & 1.15 \\
\hline $0518-458$ & 1 & $\ldots$ & GAL & 791.0 & 5.0 & $<24.5$ & 4.9 & $<3.1$ & 0.6 & $\ldots$ & 2.27 & $\ldots$ & $\ldots$ \\
\hline $0602-319$ & 1 & 0.45200 & QSO & 416.0 & 1.4 & 18.1 & 2.8 & 4.4 & 0.7 & 0.67 & 0.84 & 27.03 & 1.00 \\
\hline 0604-203 & 2 & 0.16400 & GAL & 172.5 & 1.4 & 13.3 & 2.3 & 7.7 & 1.3 & 0.81 & 1.37 & 26.07 & 0.40 \\
\hline 0614-349 & 1 & 0.32900 & GAL & 494.1 & 1.7 & 17.8 & 4.0 & 3.6 & 0.8 & 0.58 & 0.77 & 26.78 & $<0.08$ \\
\hline $0806-103$ & 2 & 0.10700 & GAL & 388.0 & 3.0 & 18.4 & 4.7 & 4.7 & 1.2 & 0.40 & 1.10 & 25.87 & 4.10 \\
\hline 0834-196 & 2 & $\ldots$ & GAL? & 410.0 & 1.4 & 12.3 & 2.8 & 3.0 & 0.7 & 0.89 & 1.00 & $\ldots$ & 0.16 \\
\hline $0842-754$ & 1 & $\ldots$ & QSO & 305.0 & 5.0 & $<26.5$ & 5.3 & $<8.7$ & 1.7 & $\ldots$ & 1.17 & $\ldots$ & $\ldots$ \\
\hline $0858-279$ & 2 & 2.15200 & QSO & 1477.0 & 1.6 & 105.5 & 6.7 & 7.1 & 0.5 & 0.02 & -0.03 & 28.15 & $<0.15$ \\
\hline 0915-118 & 2 & 0.05470 & GAL & 1851.4 & 3.3 & 222.4 & 18.8 & 12.0 & 1.0 & 0.84 & 1.54 & 26.23 & 0.04 \\
\hline 0941-080 & 2 & $\ldots$ & GAL & 371.5 & 2.1 & $<12.7$ & 2.5 & $<3.4$ & 0.7 & 0.72 & 0.82 & $\ldots$ & 0.09 \\
\hline $1015-314$ & 1 & $\ldots$ & GAL & 451.3 & 1.6 & 37.8 & 3.4 & 8.4 & 0.8 & 0.79 & 0.86 & $\ldots$ & 0.08 \\
\hline $1017-426$ & 1 & $\ldots$ & QSO & 249.9 & 0.7 & 21.2 & 1.8 & 8.5 & 0.7 & $\ldots$ & 1.24 & $\ldots$ & $\ldots$ \\
\hline $1143-483$ & 1 & $\ldots$ & GAL & 419.7 & 1.9 & $<15.8$ & 3.2 & $<3.8$ & 0.8 & $\ldots$ & 0.82 & $\ldots$ & $\ldots$ \\
\hline $1151-348$ & 1 & 0.25800 & QSO & 996.9 & 1.8 & 27.0 & 5.0 & 2.7 & 0.5 & 0.61 & 0.79 & 26.89 & $<0.04$ \\
\hline $1221-423$ & 1 & $\ldots$ & GAL & 359.5 & 1.2 & 18.9 & 1.9 & 5.3 & 0.5 & $\ldots$ & 0.80 & $\ldots$ & $\ldots$ \\
\hline $1229-021$ & 2,3 & 1.04500 & QSO & 679.7 & 2.1 & 26.2 & 6.9 & 3.8 & 1.0 & 0.33 & 0.34 & 27.60 & 0.58 \\
\hline $1239-044$ & 2,3 & 0.48000 & GAL & 186.0 & 3.0 & $<17.5$ & 3.5 & $<9.4$ & 1.9 & 1.01 & 1.29 & 27.05 & 0.45 \\
\hline $1245-197$ & 2 & 1.27500 & QSO & 767.7 & 1.6 & 16.9 & 4.3 & 2.2 & 0.6 & 0.56 & 0.90 & 28.22 & 0.33 \\
\hline $1246-410$ & 1 & $\ldots$ & GAL & 209.1 & 0.9 & 23.4 & 2.7 & 11.2 & 1.3 & $\ldots$ & 1.44 & $\ldots$ & $\ldots$ \\
\hline $1306-095$ & 2,3 & 0.46400 & GAL? & 841.0 & 3.0 & 54.5 & 13.9 & 6.5 & 1.7 & 0.61 & 0.63 & 27.24 & 0.28 \\
\hline $1308-220$ & 2 & $\ldots$ & $\mathrm{EF}$ & 215.8 & 1.3 & 21.6 & 3.4 & 10.0 & 1.6 & 0.99 & 1.28 & $\ldots$ & 0.34 \\
\hline $1318-434$ & 1 & $\ldots$ & GAL & 523.4 & 1.5 & $<12.9$ & 2.6 & $<2.5$ & 0.5 & $\ldots$ & 1.03 & $\ldots$ & $\ldots$ \\
\hline $1320-446$ & 1 & $\ldots$ & QSO & 283.1 & 0.6 & 8.9 & 1.3 & 3.2 & 0.5 & $\ldots$ & 1.01 & $\ldots$ & $\ldots$ \\
\hline $1322-428$ & 1 & $\ldots$ & GAL & 6609.9 & 6.9 & 93.0 & 3.5 & 1.4 & 0.1 & $\ldots$ & -0.60 & $\ldots$ & $\ldots$ \\
\hline $1331-098$ & 2,3 & 0.08100 & GAL & 70.0 & 4.0 & $<22.5$ & 4.5 & $<32.1$ & 6.5 & $\ldots$ & 2.25 & $\ldots$ & $\ldots$ \\
\hline $1333-337$ & 1 & 0.01290 & GAL & 269.7 & 1.7 & 16.8 & 2.3 & 6.2 & 0.9 & $\ldots$ & 2.47 & $\ldots$ & $\ldots$ \\
\hline $1335-061$ & 2,3 & 0.62500 & QSO & 146.0 & 3.0 & $<13.5$ & 2.7 & $<9.2$ & 1.9 & 0.83 & 1.48 & 27.27 & 2.24 \\
\hline $1355-416$ & 1 & $\ldots$ & QSO & 194.5 & 1.7 & 11.3 & 1.9 & 5.8 & 1.0 & $\ldots$ & 1.53 & $\ldots$ & $\ldots$ \\
\hline $1524-136$ & 2,3 & 1.68700 & QSO & 461.4 & 3.7 & 20.5 & 7.6 & 4.4 & 1.6 & 0.66 & 0.74 & 28.19 & 0.22 \\
\hline $1637-771$ & 1 & $\ldots$ & GAL & 430.8 & 1.7 & $<18.0$ & 3.6 & $<4.2$ & 0.8 & $\ldots$ & 1.44 & $\ldots$ & $\ldots$ \\
\hline $1733-565$ & 1 & $\ldots$ & GAL & 342.5 & 1.7 & 14.3 & 2.4 & 4.2 & 0.7 & $\ldots$ & 1.76 & $\ldots$ & $\ldots$ \\
\hline
\end{tabular}


Table 5. continued.

\begin{tabular}{|c|c|c|c|c|c|c|c|c|c|c|c|c|c|}
\hline$\overline{(1)}$ & (2) & (3) & (4) & $(5)$ & (6) & (7) & $(8)$ & (9) & (10) & $\overline{(11)}$ & $(12)$ & (13) & $\overline{(14)}$ \\
\hline name & dates & $z$ & type & $I$ & $\sigma_{I}$ & $F_{\mathrm{p}}$ & $\sigma_{\mathrm{F}}$ & $\Pi_{18.5}$ & $\sigma_{\Pi}$ & $\alpha_{1.4}^{5}$ & $\alpha_{5}^{18.5}$ & $\log L_{5}$ & $\Pi_{1.4}$ \\
\hline $1740-517$ & 1 & $\ldots$ & GAL? & 1361.8 & 1.6 & 16.5 & 3.1 & 1.2 & 0.2 & $\ldots$ & 0.61 & $\ldots$ & $\ldots$ \\
\hline $1814-637$ & 1 & $\ldots$ & GAL & 1584.3 & 2.2 & $<13.0$ & 2.6 & $<0.8$ & 0.2 & $\ldots$ & 0.79 & $\ldots$ & $\ldots$ \\
\hline $1829-718$ & 1 & $\ldots$ & NO ID & 258.3 & 0.5 & 10.0 & 1.2 & 3.9 & 0.4 & $\ldots$ & 1.05 & $\ldots$ & $\ldots$ \\
\hline $1934-638$ & $1,2,3$ & $\ldots$ & GAL & 1166.3 & 0.3 & 8.6 & 0.5 & 0.7 & 0.0 & $\ldots$ & 1.28 & $\ldots$ & $\ldots$ \\
\hline $1938-155$ & 3 & 0.45200 & GAL & 495.1 & 7.2 & 39.9 & 9.0 & 8.1 & 1.8 & 0.86 & 1.15 & 27.32 & 3.32 \\
\hline 2008-068 & 3 & $\ldots$ & GAL & 399.7 & 6.2 & 28.5 & 7.0 & 7.1 & 1.8 & 0.50 & 0.94 & .. & 0.28 \\
\hline $2032-350$ & 1 & $\ldots$ & GAL? & 468.0 & 2.0 & 17.9 & 6.0 & 3.8 & 1.3 & 0.84 & 1.08 & $\ldots$ & 5.75 \\
\hline 2044-027 & 3 & 0.94200 & QSO & 382.0 & 6.0 & $<27.0$ & 5.4 & $<7.1$ & 1.4 & 0.63 & 0.75 & 27.58 & 0.35 \\
\hline $2053-201$ & 3 & 0.15600 & GAL & 109.0 & 7.0 & 38.4 & 8.1 & 35.2 & 7.5 & 0.77 & 1.70 & 26.01 & $<0.14$ \\
\hline 2135-209 & 3 & 0.63500 & GAL & 479.9 & 7.1 & 39.4 & 10.0 & 8.2 & 2.1 & 0.69 & 0.89 & 27.43 & $<0.06$ \\
\hline $2140-816$ & 1 & $\ldots$ & NO ID & 149.8 & 1.4 & 12.3 & 1.9 & 8.2 & 1.3 & ... & 1.451 & $\ldots$ & $\ldots$ \\
\hline 2149-287 & 3 & $\ldots$ & NO ID & 403.6 & 8.1 & 43.3 & 9.4 & 10.7 & 2.3 & 0.585 & 0.929 & $\ldots$ & 2.67 \\
\hline $2150-520$ & 1 & $\ldots$ & QSO? & 268.9 & 0.6 & 11.2 & 1.8 & 4.2 & 0.7 & $\ldots$ & 1.150 & .. & ... \\
\hline $2226-411$ & 1 & $\ldots$ & QSO & 295.2 & 0.8 & 25.4 & 1.5 & 8.6 & 0.5 & $\ldots$ & 0.991 & $\ldots$ & ... \\
\hline $2250-412$ & 1 & $\ldots$ & GAL & 281.3 & 0.7 & 13.7 & 2.0 & 4.9 & 0.7 & $\ldots$ & 1.187 & $\ldots$ & $\ldots$ \\
\hline $2252-530$ & 1 & $\ldots$ & GAL? & 279.2 & 0.6 & 17.5 & 1.3 & 6.3 & 0.5 & $\ldots$ & 0.983 & $\ldots$ & $\ldots$ \\
\hline $2317-277$ & 2,3 & $\ldots$ & GAL & 67.0 & 2.0 & $<32.0$ & 6.4 & $<47.8$ & 9.6 & 0.398 & 2.267 & $\ldots$ & 16.20 \\
\hline $2323-407$ & 1 & $\ldots$ & GAL & 221.5 & 0.8 & 40.5 & 1.5 & 18.3 & 0.7 & $\ldots$ & 1.218 & $\cdots$ & $\cdots$ \\
\hline $2331-417$ & 1 & $\ldots$ & GAL & 290.0 & 1.4 & 12.8 & 2.3 & 4.4 & 0.8 & $\ldots$ & 1.301 & $\ldots$ & $\ldots$ \\
\hline
\end{tabular}

Table 6. Summary of observed and derived quantities for flat-spectrum sources. Same columns as in Table 5.

\begin{tabular}{|c|c|c|c|c|c|c|c|c|c|c|c|c|c|}
\hline (1) & (2) & (3) & (4) & (5) & (6) & (7) & (8) & (9) & (10) & (11) & (12) & (13) & $\overline{(14)}$ \\
\hline name & dates & $z$ & type & $I$ & $\sigma_{I}$ & $F_{\mathrm{p}}$ & $\sigma_{\mathrm{F}}$ & $\Pi_{18.5}$ & $\sigma_{\Pi}$ & $\alpha_{1.4}^{5}$ & $\alpha_{5}^{18.5}$ & $\log L_{5}$ & $\Pi_{1.4}$ \\
\hline $0003-066$ & 2,3 & 0.34700 & $\overline{Q S O}$ & 2561.3 & 11.9 & 29.8 & 13.4 & 1.2 & 0.5 & 0.25 & -0.41 & 26.82 & 2.7 \\
\hline $0047-579$ & 1 & 1.79700 & QSO & 1643.0 & 2.8 & 77.5 & 7.8 & 4.7 & 0.5 & $\ldots$ & 0.29 & $\ldots$ & $\ldots$ \\
\hline 0048-097 & 2,3 & $\ldots$ & QSO & 1397.1 & 4.9 & 37.2 & 7.7 & 2.7 & 0.5 & -0.69 & 0.26 & $\ldots$ & 3.1 \\
\hline $0056-572$ & 1 & $\ldots$ & QSO & 580.1 & 3.1 & $<20.1$ & 4.0 & $<3.5$ & 0.7 & $\cdots$ & 0.48 & $\ldots$ & $\ldots$ \\
\hline $0112-017$ & 2,3 & 1.38100 & QSO & 777.6 & 5.4 & 30.5 & 6.9 & 3.9 & 0.9 & -0.08 & 0.33 & 27.72 & 1.0 \\
\hline 0113-118 & 2,3 & 0.67200 & QSO & 1430.1 & 2.9 & 28.4 & 7.6 & 2.0 & 0.5 & -0.06 & 0.23 & 27.41 & 3.9 \\
\hline $0122-003$ & 2,3 & 1.07000 & GAL & 1721.5 & 3.4 & 60.0 & 10.7 & 3.5 & 0.6 & 0.17 & -0.25 & 27.63 & 4.4 \\
\hline $0130-171$ & 2,3 & 1.02200 & QSO & 1614.4 & 8.4 & 36.7 & 6.8 & 2.3 & 0.4 & -0.14 & -0.36 & 27.41 & 1.3 \\
\hline $0131-522$ & 1 & $\ldots$ & QSO & 794.5 & 1.8 & 24.7 & 4.5 & 3.1 & 0.6 & $\ldots$ & 0.32 & $\ldots$ & $\ldots$ \\
\hline 0138-097 & 2,3 & 0.50100 & QSO & 922.8 & 3.0 & 59.4 & 11.3 & 6.4 & 1.2 & -0.48 & 0.21 & $\ldots$ & 1.5 \\
\hline $0202-172$ & 2 & 1.74000 & QSO & 1087.3 & 4.8 & $<20.3$ & 4.1 & $<1.9$ & 0.4 & -0.09 & 0.18 & 27.93 & 2.6 \\
\hline $0208-512$ & 1 & 1.00300 & BL/QSO & 2913.7 & 7.1 & 99.2 & 9.9 & 3.4 & 0.3 & - & 0.09 & $\ldots$ & $\ldots$ \\
\hline $0238-084$ & 2,3 & 0.00500 & QSO & 1012.5 & 8.2 & $<24.4$ & 4.9 & $<2.4$ & 0.5 & -0.35 & 0.26 & $\ldots$ & 0.3 \\
\hline $0302-623$ & 1 & $\ldots$ & QSO & 1870.5 & 2.4 & 61.8 & 10.9 & 3.3 & 0.6 & $\ldots$ & -0.21 & $\ldots$ & $\ldots$ \\
\hline $0308-611$ & 1 & $\ldots$ & QSO & 1215.5 & 1.0 & 18.9 & 4.1 & 1.6 & 0.3 & $\ldots$ & 0.08 & $\cdots$ & $\cdots$ \\
\hline $0332-403$ & 1 & 1.44500 & QSO & 1786.4 & 1.9 & 63.9 & 9.1 & 3.6 & 0.5 & $\ldots$ & 0.27 & $\ldots$ & $\ldots$ \\
\hline 0336-019 & 2,3 & 0.85200 & QSO & 4485.9 & 7.8 & 147.1 & 21.7 & 3.3 & 0.5 & -0.13 & -0.34 & 27.74 & 3.2 \\
\hline $0400-319$ & 1 & $\ldots$ & GAL & 885.6 & 3.2 & 22.0 & 5.8 & 2.5 & 0.7 & -0.34 & 0.13 & $\ldots$ & 1.8 \\
\hline $0402-362$ & 1 & 1.41700 & QSO & 3180.9 & 3.0 & 42.2 & 4.1 & 1.3 & 0.1 & -0.14 & -0.63 & 27.78 & 0.4 \\
\hline $0403-132$ & 2 & 0.57100 & QSO & 2292.2 & 3.0 & 36.6 & 5.6 & 1.6 & 0.2 & 0.30 & 0.17 & 27.53 & 2.1 \\
\hline $0405-385$ & 1 & 1.28500 & QSO & 1636.5 & 2.9 & 24.9 & 5.7 & 1.5 & 0.3 & -0.18 & -0.31 & 27.59 & 1.1 \\
\hline $0405-123$ & 2 & 0.57400 & BL/QSO & 1430.6 & 3.3 & 23.8 & 5.8 & 1.7 & 0.4 & 0.31 & 0.24 & 27.37 & 1.1 \\
\hline 0414-189 & 2 & 1.53600 & QSO & 913.0 & 2.8 & 25.1 & 4.4 & 2.7 & 0.5 & -0.06 & 0.29 & 27.85 & 0.5 \\
\hline 0420-014 & 2 & 0.91500 & QSO & 7912.8 & 13.0 & 85.5 & 12.5 & 1.1 & 0.2 & 0.49 & -1.29 & 27.67 & 1.2 \\
\hline 0426-380 & 1 & 1.03000 & BL/QSO & 1056.3 & 2.2 & 61.5 & 6.2 & 5.8 & 0.6 & -0.36 & 0.07 & $\ldots$ & 7.0 \\
\hline 0434-188 & 2 & 2.70200 & QSO & 423.9 & 2.1 & 13.7 & 3.6 & 3.2 & 0.8 & -0.42 & 0.81 & 27.98 & 0.7 \\
\hline $0437-454$ & 1 & $\ldots$ & QSO & 1127.2 & 1.4 & 36.5 & 5.1 & 3.2 & 0.5 & $\ldots$ & 0.17 & $\ldots$ & $\ldots$ \\
\hline $0438-436$ & 1 & 2.85200 & QSO & 3926.9 & 3.0 & 97.3 & 2.4 & 2.5 & 0.1 & $\cdots$ & 0.43 & $\ldots$ & $\ldots$ \\
\hline 0440-003 & 2 & 0.84400 & QSO & 1214.8 & 3.4 & 22.6 & 6.4 & 1.9 & 0.5 & -0.30 & 0.58 & 27.65 & 0.6 \\
\hline $0454-810$ & 1 & 0.44400 & QSO & 1574.3 & 3.5 & 40.7 & 7.3 & 2.6 & 0.5 & $\ldots$ & -0.09 & $\ldots$ & ... \\
\hline $0451-282$ & 2 & 2.55900 & QSO & 1746.6 & 1.6 & 46.9 & 7.8 & 2.7 & 0.4 & 0.09 & 0.19 & 28.50 & 0.8 \\
\hline $0454-463$ & 1 & 0.85800 & QSO & 3599.6 & 2.3 & 27.8 & 3.8 & 0.8 & 0.1 & $\cdots$ & -0.33 & $\cdots$ & $\ldots$ \\
\hline $0454-234$ & 2 & 1.00300 & QSO & 5389.8 & 4.9 & 157.5 & 4.3 & 2.9 & 0.1 & -0.13 & -0.73 & 27.71 & 3.2 \\
\hline
\end{tabular}


Table 6. continued.

\begin{tabular}{|c|c|c|c|c|c|c|c|c|c|c|c|c|c|}
\hline$\overline{(1)}$ & $\overline{(2)}$ & (3) & $\overline{(4)}$ & $\overline{(5)}$ & $\overline{(6)}$ & $\overline{(7)}$ & $\overline{(8)}$ & $\overline{(9)}$ & (10) & $\overline{(11)}$ & $\overline{(12)}$ & $\overline{(13)}$ & $\overline{(14)}$ \\
\hline name & dates & $z$ & type & $I$ & $\sigma_{I}$ & $F_{\mathrm{p}}$ & $\sigma_{\mathrm{F}}$ & $\Pi_{18.5}$ & $\sigma_{\Pi}$ & $\alpha_{1.4}^{5}$ & $\alpha_{5}^{18.5}$ & $\log L_{5}$ & $\Pi_{1.4}$ \\
\hline $0458-020$ & 2 & 2.28600 & QSO & 1568.7 & 3.5 & 24.5 & 6.8 & 1.6 & 0.4 & 0.20 & 0.07 & 28.37 & 0.5 \\
\hline $0506-612$ & 1 & 1.09300 & BL/QSO & 2436.4 & 2.0 & 50.2 & 9.5 & 2.1 & 0.4 & 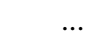 & -0.26 & 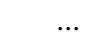 & 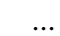 \\
\hline $0511-220$ & 2 & 1.29600 & QSO & 768.5 & 2.2 & 34.2 & 5.7 & 4.5 & 0.7 & -0.55 & 0.40 & 27.54 & 0.9 \\
\hline 0514-459 & 1 & 0.19400 & QSO & 886.8 & 2.1 & 35.9 & 7.1 & 4.0 & 0.8 & $\ldots$ & 0.13 & $\ldots$ & ... \\
\hline $0524-460$ & 1 & 1.47900 & QSO & 523.9 & 2.6 & 15.9 & 3.3 & 3.0 & 0.6 & $\ldots$ & 0.50 & $\ldots$ & $\ldots$ \\
\hline $0528-250$ & 2 & 2.76500 & QSO & 469.3 & 2.7 & 14.4 & 2.7 & 3.1 & 0.6 & 0.00 & 0.69 & 28.21 & 0.6 \\
\hline $0537-441$ & 1 & 0.89600 & QSO & 10682.9 & 3.9 & 290.8 & 2.7 & 2.7 & 0.0 & $\ldots$ & -0.75 & $\ldots$ & $\ldots$ \\
\hline $0537-286$ & 2 & 3.11900 & QSO & 865.7 & 3.0 & 20.1 & 3.9 & 2.3 & 0.5 & -0.13 & 0.12 & 28.16 & 0.6 \\
\hline 0539-057 & 2 & 0.83900 & QSO & 782.7 & 2.8 & 21.0 & 4.1 & 2.7 & 0.5 & -0.44 & 0.52 & 27.38 & 4.3 \\
\hline $0605-085$ & 2 & 0.87200 & BL/QSO & 1958.8 & 3.2 & 58.6 & 2.8 & 3.0 & 0.1 & -0.47 & 0.44 & 27.75 & 2.2 \\
\hline $0606-223$ & 2 & 1.92600 & QSO & 949.1 & 1.8 & 23.6 & 4.6 & 2.5 & 0.5 & -0.56 & 0.29 & 27.79 & 1.9 \\
\hline $0607-157$ & 2 & 0.32400 & QSO & 5999.5 & 7.5 & 287.2 & 5.1 & 4.8 & 0.1 & 0.32 & -0.91 & 26.86 & 1.3 \\
\hline $0637-752$ & 1 & 0.65400 & QSO & 4777.8 & 4.4 & 156.8 & 3.0 & 3.3 & 0.1 & $\ldots$ & 0.15 & $\ldots$ & $\ldots$ \\
\hline $0642-349$ & 1 & 2.16500 & QSO & 321.5 & 2.1 & 16.5 & 3.0 & 5.1 & 0.9 & -0.27 & 0.88 & 27.86 & 1.0 \\
\hline 0743-006 & 2 & 0.99400 & QSO & 1615.1 & 3.7 & 35.1 & 5.1 & 2.2 & 0.3 & -0.86 & 0.16 & 27.47 & 0.4 \\
\hline 0805-077 & 2 & 1.83700 & QSO & 1659.5 & 2.0 & 21.0 & 6.2 & 1.3 & 0.4 & 0.33 & -0.35 & 28.04 & 1.4 \\
\hline 0834-201 & 2 & 2.75200 & QSO & 4383.0 & 5.8 & 81.7 & 3.9 & 1.9 & 0.1 & -0.49 & -0.12 & 28.43 & 0.3 \\
\hline $0859-140$ & 2 & 1.33900 & QSO & 1190.3 & 1.5 & 38.4 & 3.5 & 3.2 & 0.3 & 0.18 & 0.50 & 28.08 & 3.2 \\
\hline 0919-260 & 2 & 2.30000 & QSO & 1442.5 & 2.1 & 23.0 & 4.7 & 1.6 & 0.3 & -0.01 & -0.06 & 28.14 & 0.9 \\
\hline 1032-199 & 2 & 2.19800 & QSO & 1260.3 & 2.4 & 26.7 & 6.5 & 2.1 & 0.5 & -0.18 & -0.07 & 27.97 & 5.3 \\
\hline $1045-188$ & 2 & 0.59500 & QSO & 1625.2 & 2.1 & 36.4 & 7.7 & 2.2 & 0.5 & 0.01 & -0.27 & 27.10 & 2.3 \\
\hline $1057-797$ & 1 & $\ldots$ & QSO & 2638.0 & 1.9 & 100.1 & 8.4 & 3.8 & 0.3 & $\ldots$ & -0.37 & $\ldots$ & $\ldots$ \\
\hline $1104-445$ & 1 & 1.59800 & QSO & 2273.0 & 2.4 & 27.7 & 3.5 & 1.2 & 0.2 & $\ldots$ & -0.07 & $\cdots$ & $\ldots$ \\
\hline $1116-462$ & 1 & 0.71300 & BL/QSO & 963.8 & 2.3 & 39.8 & 1.3 & 4.1 & 0.1 & $\ldots$ & 0.25 & $\cdots$ & $\ldots$ \\
\hline $1127-145$ & 2 & 1.18700 & QSO & 2926.2 & 2.2 & 116.3 & 2.7 & 4.0 & 0.1 & -0.12 & 0.61 & 28.34 & 4.0 \\
\hline $1143-245$ & 2 & 1.95000 & QSO & 691.3 & 3.3 & $<17.7$ & 3.5 & $<2.6$ & 0.5 & 0.15 & 0.40 & 28.06 & 1.7 \\
\hline 1144-379 & 1 & 1.04800 & QSO & 4431.6 & 5.8 & 323.7 & 2.2 & 7.3 & 0.0 & 0.08 & -0.77 & $\cdots$ & 2.2 \\
\hline $1145-071$ & 2 & 1.34200 & QSO & 809.9 & 1.7 & 22.2 & 3.6 & 2.7 & 0.4 & -0.41 & 0.33 & 27.60 & 1.9 \\
\hline $1148-001$ & 2 & 1.98200 & QSO & 972.2 & 1.0 & 20.6 & 4.6 & 2.1 & 0.5 & 0.29 & 0.51 & 28.35 & 4.6 \\
\hline $1202-262$ & 2 & 0.78900 & NO ID & 509.3 & 1.5 & $<11.5$ & 2.3 & $<2.3$ & 0.5 & 0.36 & 0.54 & 27.37 & 1.1 \\
\hline $1213-172$ & 2 & $\ldots$ & GAL & 1897.1 & 2.5 & 41.3 & 3.9 & 2.2 & 0.2 & 0.09 & -0.19 & $\cdots$ & 1.1 \\
\hline $1237-101$ & 2,3 & 0.75300 & QSO & 1032.6 & 5.4 & 23.6 & 7.6 & 2.3 & 0.7 & 0.11 & 0.18 & 27.37 & 2.9 \\
\hline $1243-072$ & 2,3 & 1.28600 & QSO & 712.1 & 1.0 & 24.5 & 6.7 & 3.4 & 0.9 & $\ldots$ & 0.28 & & $\ldots$ \\
\hline $1244-255$ & 2 & 0.63300 & GAL & 1848.1 & 3.1 & 60.6 & 14.4 & 3.3 & 0.8 & -0.12 & -0.23 & 27.20 & 3.6 \\
\hline $1253-055$ & $1,2,3$ & 0.53600 & QSO & 28043.5 & 12.3 & 1648.5 & 1.7 & 5.9 & 0.0 & -0.33 & -0.48 & 28.07 & 2.6 \\
\hline $1255-316$ & 1 & 1.92400 & QSO & 2000.6 & 3.5 & 86.9 & 13.7 & 4.3 & 0.7 & -0.32 & -0.11 & 27.99 & 5.3 \\
\hline $1302-102$ & 2,3 & 0.28600 & QSO & 827.0 & 2.0 & 26.4 & 7.4 & 3.2 & 0.9 & -0.39 & 0.26 & 26.48 & 0.4 \\
\hline $1313-333$ & 1 & 1.21000 & BL/QSO & 1457.6 & 3.0 & 33.2 & 8.0 & 2.3 & 0.5 & -0.04 & -0.05 & 27.69 & 1.1 \\
\hline $1334-127$ & 2,3 & 0.53900 & QSO & 7107.4 & 6.8 & 88.4 & 23.0 & 1.2 & 0.3 & 0.14 & -0.88 & 27.34 & 2.3 \\
\hline $1352-104$ & 2,3 & 0.33200 & QSO & 1659.4 & 2.0 & 35.1 & 10.9 & 2.1 & 0.7 & -0.21 & -0.37 & 26.56 & 4.3 \\
\hline $1354-152$ & 2 & 1.89000 & QSO & 999.8 & 1.3 & 21.4 & 6.0 & 2.1 & 0.6 & -0.63 & 0.32 & 27.78 & 2.0 \\
\hline $1406-076$ & 2,3 & 1.49400 & QSO & 1792.3 & 4.9 & 35.2 & 11.3 & 2.0 & 0.6 & -0.35 & -0.38 & 27.62 & 3.1 \\
\hline $1424-418$ & 1 & 1.52200 & QSO & 2923.3 & 2.1 & 25.6 & 4.5 & 0.9 & 0.2 & $\ldots$ & 0.05 & & $\cdots$ \\
\hline $1451-375$ & 1 & 0.31400 & QSO & 2004.8 & 3.1 & 78.1 & 5.8 & 3.9 & 0.3 & -0.54 & -0.04 & 26.75 & 3.0 \\
\hline $1504-166$ & 3 & 0.87600 & QSO & 1498.6 & 10.0 & $<47.3$ & 9.5 & $<3.2$ & 0.6 & 0.24 & 0.21 & 27.70 & 0.3 \\
\hline $1508-055$ & 2,3 & 1.19100 & BL/QSO & 1272.2 & 6.2 & 37.3 & 11.9 & 2.9 & 0.9 & 0.30 & 0.49 & 28.05 & 1.2 \\
\hline $1510-089$ & 2,3 & 0.36100 & QSO & 2527.6 & 5.7 & 63.1 & 18.9 & 2.5 & 0.7 & -0.10 & 0.15 & 27.12 & 3.4 \\
\hline $1514-241$ & 3 & 0.04860 & QSO & 2030.1 & 11.4 & 86.9 & 13.4 & 4.3 & 0.7 & 0.01 & -0.01 & $\ldots$ & 5.1 \\
\hline $1519-273$ & 3 & $\ldots$ & QSO & 1632.3 & 8.8 & 90.4 & 6.8 & 5.5 & 0.4 & -0.59 & 0.27 & $\ldots$ & 1.9 \\
\hline $1541-828$ & 1 & & GAL & 589.7 & 1.8 & 15.0 & 3.1 & 2.5 & 0.5 & $\ldots$ & 0.69 & $\ldots$ & $\ldots$ \\
\hline $1549-790$ & 1 & 0.14900 & QSO & 1429.2 & 2.1 & $<14.8$ & 3.0 & $<1.0$ & 0.2 & $\ldots$ & 0.72 & $\ldots$ & $\ldots$ \\
\hline $1555+001$ & 2,3 & 1.77000 & QSO & 891.5 & 3.8 & $<26.3$ & 5.3 & $<3.0$ & 0.6 & -0.55 & 0.70 & 27.95 & 0.9 \\
\hline $1610-771$ & 1 & 1.71000 & QSO & 2674.3 & 3.0 & 46.1 & 2.0 & 1.7 & 0.1 & $\ldots$ & 0.29 & $\ldots$ & $\ldots$ \\
\hline $1619-680$ & 1 & 1.35400 & GAL & 724.7 & 1.4 & $<14.7$ & 2.9 & $<2.0$ & 0.4 & $\cdots$ & 0.72 & $\cdots$ & $\cdots$ \\
\hline $1622-253$ & 3 & & QSO & 2144.1 & 19.4 & 86.7 & 16.8 & 4.0 & 0.8 & 0.15 & -0.02 & $\cdots$ & 0.5 \\
\hline $1718-649$ & 1 & 0.01450 & QSO & 3003.2 & 2.7 & 18.7 & 4.4 & 0.6 & 0.1 & $\ldots$ & 0.18 & 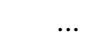 & ... \\
\hline
\end{tabular}


Table 6. continued.

\begin{tabular}{|c|c|c|c|c|c|c|c|c|c|c|c|c|c|}
\hline$\overline{(1)}$ & $\overline{(2)}$ & (3) & $\overline{(4)}$ & $\overline{(5)}$ & (6) & $\overline{(7)}$ & $\overline{(8)}$ & $\overline{(9)}$ & (10) & $\overline{(11)}$ & $\overline{(12)}$ & $\overline{(13)}$ & $\overline{(14)}$ \\
\hline name & dates & $z$ & type & $I$ & $\sigma_{I}$ & $F_{\mathrm{p}}$ & $\sigma_{\mathrm{F}}$ & $\Pi_{18.5}$ & $\sigma_{\Pi}$ & $\alpha_{1.4}^{5}$ & $\alpha_{5}^{18.5}$ & $\log L_{5}$ & $\Pi_{1.4}$ \\
\hline $1741-038$ & 3 & 1.05700 & QSO & 4921.9 & 21.8 & 77.2 & 13.6 & 1.6 & 0.3 & -0.75 & -0.22 & 27.81 & $\overline{0.6}$ \\
\hline $1815-553$ & 1 & $\ldots$ & QSO & 1097.2 & 2.2 & 15.4 & 3.4 & 1.4 & 0.3 & $\ldots$ & 0.15 & $\ldots$ & $\ldots$ \\
\hline $1831-711$ & 1 & 1.35600 & QSO & 2221.1 & 2.1 & 27.1 & 4.3 & 1.2 & 0.2 & $\ldots$ & -0.47 & $\ldots$ & ... \\
\hline $1903-802$ & 1 & 0.50000 & QSO & 614.9 & 2.0 & 18.5 & 3.5 & 3.0 & 0.6 & $\ldots$ & 0.83 & $\ldots$ & ... \\
\hline $1933-400$ & 1 & 0.96600 & QSO & 1162.7 & 1.5 & 26.9 & 3.3 & 2.3 & 0.3 & -0.30 & 0.18 & 27.49 & 2.8 \\
\hline $1936-155$ & 3 & 1.65700 & QSO & 966.6 & 8.1 & 34.7 & 9.4 & 3.6 & 1.0 & -0.80 & 0.42 & 27.69 & 0.8 \\
\hline $1936-623$ & 1 & $\ldots$ & QSO & 531.4 & 1.4 & 24.4 & 4.0 & 4.6 & 0.8 & $\ldots$ & 0.55 & $\ldots$ & $\ldots$ \\
\hline $1954-388$ & 1 & 0.62600 & GAL & 3810.4 & 1.8 & 26.5 & 6.0 & 0.7 & 0.2 & -0.25 & -0.47 & 27.34 & 1.3 \\
\hline $1958-179$ & 3 & 0.65000 & QSO & 1337.7 & 6.0 & 39.7 & 8.7 & 3.0 & 0.6 & -0.61 & -0.08 & 27.06 & 0.7 \\
\hline $2000-330$ & 1 & 3.77700 & $\mathrm{EF}$ & 514.7 & 1.9 & $<13.3$ & 2.7 & $<2.6$ & 0.5 & -0.74 & 0.61 & 27.91 & $<0.6$ \\
\hline $2005-489$ & 1 & 0.07100 & QSO & 1345.5 & 1.3 & $<15.9$ & 3.2 & $<1.2$ & 0.2 & $\ldots$ & -0.06 & $\cdots$ & $\cdots$ \\
\hline $2008-159$ & 3 & 1.18000 & QSO & 2245.9 & 7.5 & 46.7 & 9.3 & 2.1 & 0.4 & -0.73 & -0.36 & 27.45 & 1.3 \\
\hline $2052-474$ & 1 & 1.49100 & QSO & 739.1 & 1.5 & 12.3 & 2.8 & 1.7 & 0.4 & $\ldots$ & 0.93 & $\ldots$ & ... \\
\hline 2106-413 & 1 & 1.05470 & QSO & 1895.0 & 2.8 & 29.7 & 4.5 & 1.6 & 0.2 & $\ldots$ & 0.16 & $\ldots$ & ... \\
\hline $2126-158$ & 3 & 3.26600 & QSO & 933.5 & 10.9 & 36.9 & 9.5 & 4.0 & 1.0 & -0.60 & 0.24 & 27.98 & $<0.4$ \\
\hline $2128-123$ & 3 & 0.50100 & QSO & 3390.8 & 16.8 & 86.6 & 21.7 & 2.6 & 0.6 & -0.12 & -0.37 & 27.20 & 2.0 \\
\hline $2131-021$ & 3 & 0.55700 & QSO & 2272.8 & 17.9 & 79.7 & 20.0 & 3.5 & 0.9 & -0.17 & -0.05 & $\ldots$ & 1.2 \\
\hline $2142-758$ & 1 & 1.13900 & QSO & 687.9 & 2.2 & 68.4 & 10.5 & 9.9 & 1.5 & $\cdots$ & 0.49 & $\ldots$ & $\ldots$ \\
\hline $2155-152$ & 3 & 0.67200 & QSO & 2505.5 & 17.1 & 60.5 & 14.7 & 2.4 & 0.6 & 0.42 & -0.26 & 27.48 & 4.0 \\
\hline $2203-188$ & 2,3 & 0.61900 & QSO & 2200.0 & 11.6 & 85.8 & 11.5 & 3.9 & 0.5 & 0.29 & 0.52 & 27.78 & 0.2 \\
\hline $2204-540$ & 1,3 & 1.20600 & QSO & 1306.6 & 2.2 & 86.1 & 2.0 & 6.6 & 0.2 & $\ldots$ & 0.46 & $\ldots$ &. \\
\hline $2206-237$ & 2,3 & 0.08700 & QSO & 340.6 & 2.5 & 24.6 & 3.8 & 7.2 & 1.1 & 0.53 & 0.83 & $\cdots$ & 0.2 \\
\hline $2210-257$ & 2,3 & 1.83300 & QSO & 609.1 & 15.2 & 29.3 & 7.6 & 4.8 & 1.3 & 0.11 & 0.41 & 27.94 & 1.4 \\
\hline $2216-038$ & 3 & 0.90100 & QSO & 2571.6 & 13.5 & 66.9 & 17.5 & 2.6 & 0.7 & 0.30 & -0.41 & 27.62 & 0.7 \\
\hline $2223-052$ & 3 & 1.40400 & QSO & 8394.5 & 10.8 & 359.7 & 10.8 & 4.3 & 0.1 & 0.39 & -0.47 & 28.49 & 4.5 \\
\hline $2227-088$ & 3 & 1.56100 & QSO & 1570.5 & 8.1 & $<40.2$ & 8.0 & $<2.6$ & 0.5 & -0.47 & 0.09 & 27.81 & 1.3 \\
\hline $2243-123$ & 3 & 0.63000 & QSO & 2043.7 & 9.8 & 48.8 & 16.4 & 2.4 & 0.8 & -0.20 & 0.13 & 27.43 & 1.4 \\
\hline $2345-167$ & 2,3 & 0.57600 & QSO & 2747.7 & 5.1 & 46.8 & 5.4 & 1.7 & 0.2 & -0.25 & 0.21 & 27.53 & 2.8 \\
\hline $2353-686$ & 1,3 & 1.71600 & QSO & 785.4 & 0.9 & 16.4 & 3.4 & 2.1 & 0.4 & $\cdots$ & 0.25 & $\cdots$ & $\cdots$ \\
\hline $2354-117$ & 3 & 0.96000 & QSO & 1197.1 & 7.2 & 54.0 & 12.3 & 4.5 & 1.0 & 0.16 & 0.16 & 27.62 & 1.5 \\
\hline $2355-534$ & 1,3 & 1.00600 & QSO & 1602.1 & 2.7 & 51.9 & 6.9 & 3.2 & 0.4 & $\ldots$ & -0.04 & $\ldots$ & $\cdots$ \\
\hline $2255-282$ & 2,3 & 0.92600 & QSO & 11226.5 & 20.3 & 379.2 & 6.7 & 3.4 & 0.1 & -0.27 & -1.40 & 27.55 & 1.0 \\
\hline $2311-452$ & 1,3 & 2.88400 & QSO & 585.6 & 1.6 & 28.1 & 4.5 & 4.8 & 0.8 & ... & 0.70 & $\cdots$ & $\cdots$ \\
\hline $2326-477$ & 1,3 & 1.30600 & GAL & 1571.0 & 2.8 & 23.1 & 3.6 & 1.5 & 0.2 & $\ldots$ & 0.30 & $\ldots$ & ... \\
\hline $2329-162$ & 2,3 & 1.15500 & QSO & 1964.4 & 8.1 & 99.2 & 19.7 & 5.0 & 1.0 & 0.18 & -0.47 & 27.63 & 1.1 \\
\hline $2331-240$ & 2,3 & 0.04770 & QSO & 1095.7 & 3.5 & 25.8 & 5.8 & 2.4 & 0.5 & -0.26 & -0.00 & $\cdots$ & 1.0 \\
\hline $2333-528$ & 1,3 & $\ldots$ & QSO & 1113.9 & 0.9 & 23.3 & 3.3 & 2.1 & 0.3 & $\ldots$ & 0.07 & $\ldots$ & $\cdots$ \\
\hline
\end{tabular}

\section{References}

Aller, M. F., Aller, H. D., Hughes, P. A., \& Latimer, G. E. 1999, ApJ, 512,601

Condon, J. J., Cotton, W. D., Greisen, E. W., et al. 1998, AJ, 115, 1693

De Zotti, G., Gruppioni, C., Ciliegi, P., Burigana, C., \& Danese, L. 1999, NewA, 4, 481

Feigelson, E. D., \& Nelson, P. I., 1985, ApJ, 293, 192

Isobe, T., \& Feigelson, E. D., 1990, BAAS, 22, 917

Isobe, T., Feigelson, E. D., \& Nelson, P. I., 1986, ApJ, 306, 490

Jones, T. W., Rudnick, L., Aller, H. D., et al. 1985, ApJ, 290, 627

Klein, U., Mack, K.-H., Gregorini, L., \& Vigotti, M., 2003, A\&A, 406, 579

Kogut, A., Spergel, D. N., Barnes, C., et al. 2003, ApJS, 148, 161

Kovac, J. M., Leitch, E. M., Pryke, C., et al. 2002, Nature, 420, 772
Kühr, H., Witzel, A., Pauliny-Toth, I. I. K., \& Nauber U. 1981, A\&AS, 45, 367

Mesa, D., Baccigalupi, C., De Zotti, G., et al. 2002, A\&A, 396, 463

Nartallo, R., Gear, W. K., Murray, A. G., Robson, E. I., \& Hough, J. H. 1998, MNRAS, 297, 667

Okudaira, A., Tabara, H., Kato, T., \& Inoue, M. 1993, PASJ, 45, 153

Pentericci, L., Van Reeven, W., Carilli, C. L., Röttgering, H. J. A., \& Miley, G. K. 2000, A\&AS, 145, 121

Rudnick, L., Jones, T. W., Aller, H. D., et al. 1985, ApJS. 57, 693

Saikia, D. J., \& Salter, C. J. 1988, ARA\&A, 26, 93

Sazhin, M. V., \& Korolëv, V. A. 1985, Sov. Astron. Lett., 11, 204

Sault, R. J., Teuben, P. J., \& Wright, M. C. H, A retrospective view of Miriad in Astronomical Data Analysis Software and Systems, ed. R. Shaw, H. E. Payne, \& J. J. E. Haynes, ASP Conf. Ser., 77, 433 Stickel, M., Meisenheimer, K., \& Kühr, H., 1994, A\&AS, 105, 211 